TRANSACTIONS OF THE

AMERICAN MATHEMATICAL SOCIETY

Volume 349, Number 6, June 1997, Pages 2477-2492

S 0002-9947(97)01816-3

\title{
DEGENERATIONS OF K3 SURFACES IN PROJECTIVE SPACE
}

\author{
FRANCISCO JAVIER GALLEGO AND B. P. PURNAPRAJNA
}

\begin{abstract}
The purpose of this article is to study a certain kind of numerical K3 surfaces, the so-called K3 carpets. These are double structures on rational normal scrolls with trivial dualizing sheaf and irregularity 0 . As is deduced from our study, K3 carpets can be obtained as degenerations of smooth K3 surfaces. We also study the Hilbert scheme near the locus parametrizing K3 carpets, characterizing those K3 carpets whose corresponding Hilbert point is smooth. Contrary to the case of canonical ribbons, not all K3 carpets are smooth points of the Hilbert scheme.
\end{abstract}

\section{INTRODUCTION}

This article deals with the study of K3 carpets. D. Bayer and D. Eisenbud say in $[\mathrm{BE}]$ that " $a$ ribbon" (supported on $\mathbf{P}^{1}$ inside $\mathbf{P}^{g-1}$ and with arithmetic genus $g$ ) "is the answer to the riddle: What is the limit of the canonical model of a smooth curve as the curve degenerates to a hyperelliptic curve?" Analogously one would say that a $K 3$ carpet is the answer to the riddle: What is the limit of the embedded model of a smooth polarized K3 surface as the polarized surface degenerates to a hyperelliptic polarized surface? To justify this claim we devote much of this article.

K3 carpets possess some interesting features. On the one hand there are few of them. In Section 1 we see that there is only one K3 carpet supported on a given rational normal scroll (in the same way as a canonical ribbon is a double structure on a rational normal curve, the reduced structure of a K3 carpet is a rational normal scroll). Thus one can in some sense think of the set of all K3 carpets as something discrete. On the other hand, some of them (the ones supported on "balanced" scrolls) are still general, in the sense that they are smooth points of the Hilbert scheme. Hence K3 carpets form a small class of very degenerate objects (they are nowhere reduced, and are one step more degenerate than such reduced nonnormal K3 surfaces as the unions of two (distinct) rational normal scrolls) which are nevertheless general.

Another interesting feature is that the hyperplane section of a K3 carpet is a canonical ribbon. The study of canonical ribbons has been proposed by Bayer, Eisenbud, Green and Schreyer, among others, as a means to solve the so-called Green's conjecture. Briefly, in its original form this conjecture relates the graded Betti numbers of the minimal free resolution of a canonical curve to the Clifford

Received by the editors January 11, 1996.

1991 Mathematics Subject Classification. Primary 14J10, 14J25, 14J28; Secondary 14C05, 14C34, 32G20.

We are very pleased to thank our advisor David Eisenbud for his help, patience and encouragement. We would also like to thank Andrea Bruno and Enrique Arrondo for helpful discussions and Mohan Kumar for his helpful comments and suggestions.

(C)1997 American Mathematical Society 
index of the curve (the Clifford index of a smooth curve of genus $g \geq 3$ is defined as the minimum, over all line bundles $L$ on the curve such that $\mathrm{h}^{0}(L)>1$ and $\mathrm{h}^{1}(L)>1$, of the quantity Cliff $L=\operatorname{deg} L-2\left(\mathrm{~h}^{0}(L)-1\right)$. More loosely put, the Clifford index tells us how special the most special line bundle which the curve possesses is). More precisely, one expects that the canonical bundle will satisfy the property $N_{p}$ but not the property $N_{p+1}$ iff $p$ is the Clifford index of the curve. Thus Green's conjecture generalizes classical results by Noether and Petri (cf. [ACGH]; for details on Green's conjecture see [E]). Results by Eisenbud and Green [EG] and Fong $[\mathrm{F}]$ yield that an affirmative answer to Green's conjecture in the case of canonical ribbons will imply Green's conjecture for general curves. Since K3 carpets are arithmetically Cohen-Macaulay, the Betti numbers of the minimal free resolution of a K3 carpet are the same as the Betti number of the hyperplane section, a canonical ribbon. Progress toward the computation of the minimal resolution of a K3 carpet has been made by Dave Bayer and David Eisenbud in [BE], where they compute the graded Betti numbers of a nonminimal resolution of a K3 carpet.

Our work on K3 carpets focuses on answering two questions: are these objects smoothable? and do they correspond to smooth points of the Hilbert scheme? The first question is dealt with and answered affirmatively in Section 3. To prove this result we use the idea, already introduced, that a (suitable) K3 carpet is morally the "image" of the morphism associated to a hyperelliptic linear system. To show this we use a characterization given in Section 2 which allows us to decide by induction on the dimension (cutting with a hyperplane) whether a scheme is a ribbon. We also use properties of hyperelliptic linear systems on K3 surfaces and of the moduli of K3 surfaces. We would like to point out here one difference between the case of canonical ribbons and the case of K3 carpets. While canonical ribbons can be thought as "canonical models" of hyperelliptic curves, not all K3 carpets are "models" of smooth hyperelliptic K3 surfaces. More precisely, rational normal scrolls with a rational curve with low self-intersection cannot be realized as images of morphisms associated to hyperelliptic linear systems, and hence the riddle posed before does not make sense for them. However we are able to prove that K3 carpets of this kind are smoothable by showing that they deform to more general ones.

In Section 4 we deal with the study of the Hilbert scheme near the locus of K3 carpets. Our main result is that K3 carpets supported on "balanced" scrolls are smooth points of the Hilbert scheme. Here another departure from the case of ribbons occurs. While both K3 carpets and canonical ribbons are smoothable (i.e, both belong to the component parametrizing smooth varieties in their respective Hilbert schemes), contrary to the case of canonical ribbons, not all K3 carpets are smooth points of the Hilbert scheme (some of them even belong to several components of the Hilbert scheme, as noted in Theorem 4.3).

\section{K3 CARPETS}

Conventions. Throughout this article we work over C. A rational normal scroll or simply a scroll will always mean a smooth rational normal scroll of dimension 2. We will denote by $F_{n}$ the rational ruled surface whose minimal section has self-intersection $-n$.

In this section we introduce our main objects of study, the K3 carpets, and some properties of them which we will use later in the article. We start with a couple of definitions: 
Definition 1.1 ([BE], §1). A double structure or a ribbon on a reduced connected scheme $D^{\prime}$ is a scheme $D$ equipped with an isomorphism $D^{\prime} \rightarrow D_{\text {red }}$, such that the ideal sheaf $\mathcal{I}$ of $D^{\prime}$ in $D$ satisfies $\mathcal{I}^{2}=0$ and is a line bundle on $D^{\prime}$.

Definition 1.2. K3 carpet $\tilde{S}$ is a double structure on a rational normal scroll $S$ (i.e., a double structure embedded in some $\mathbf{P}^{g}$, whose reduced structure is a rational normal scroll in $\left.\mathbf{P}^{g}\right)$ such that its dualizing sheaf $\omega_{\tilde{S}}$ is trivial and $h^{1}\left(\mathcal{O}_{\tilde{S}}\right)=0$.

An important fact about K3 carpets (which will be certainly instrumental to our proof of the main result of this article, namely, the smoothing of K3 carpets) is stated in this:

Theorem 1.3. There is a unique K3 carpet (up to multiplication by scalar) on a given rational normal scroll.

Before we prove Theorem 1.3 we need to state two lemmas which are variants of results in $[\mathrm{HV}]$. The lemmas identify the conormal bundle of the reduced structure of the K3 carpet inside the carpet itself. From them it follows that the K3 double structures on a scroll $S$ in $\mathbf{P}^{g}$ correspond to the global sections of a twist of the normal bundle of $S$. The proofs use the same ideas of [HV].

Lemma 1.4. Defining a double structure $\tilde{S}$ on a smooth subvariety $S$ of a smooth variety $Z$ is equivalent to giving a line subbundle $\mathcal{L}$ of $\mathcal{N}_{S / Z}$. This line bundle $\mathcal{L}$ is the normal bundle of $S$ in $\tilde{S}$

Proof. Let $\mathcal{L} \subseteq \mathcal{N}_{S / Z}$ be a line bundle and $\mathcal{I}=\mathcal{I}_{Z}(S)$ the ideal sheaf of $S$ in $Z$. Let $w$ be the surjective homomorphism

$$
w: \mathcal{I} \rightarrow \mathcal{I} / \mathcal{I}^{2}=\mathcal{N}_{S / Z}^{*} \rightarrow \mathcal{L}^{*} .
$$

Let $\mathcal{J}=\operatorname{ker} w$. The ideal sheaf $\mathcal{J}$ defines a subscheme $\tilde{S}$ in $Z$. From the exact sequences

$$
0 \rightarrow \mathcal{J} \rightarrow \mathcal{I} \rightarrow \mathcal{L}^{*} \rightarrow 0
$$

and

$$
\begin{aligned}
& 0 \rightarrow \mathcal{I} / \mathcal{J} \rightarrow \mathcal{O}_{Z} / \mathcal{J} \rightarrow \mathcal{O}_{Z} / \mathcal{I} \rightarrow 0 \\
& 0 \rightarrow \mathcal{L}^{*} \rightarrow\left\|_{\tilde{S}} \rightarrow\right\|_{S} \rightarrow 0
\end{aligned}
$$

we see that the ideal sheaf defining $S$ in $\tilde{S}$ is the line bundle $\mathcal{L}^{*}$. Since $\left(\mathcal{L}^{*}\right)^{2}=$ $(\mathcal{I} / \mathcal{J})^{2}=0$ by construction of $\mathcal{J}$, it follows that $\tilde{S}$ is a ribbon.

Conversely, let $\tilde{S}$ be a double structure embedded in $Z$, let $S$ be its reduced part, and let $\mathcal{I}, \mathcal{J}$ be their respective ideal sheaves in $Z$. By the definition of ribbon $\mathcal{I}^{2} \subseteq \mathcal{J}$, so $\mathcal{N}_{S / Z}=\mathcal{I} / \mathcal{I}^{2}$ surjects onto $\mathcal{I} / \mathcal{J}$, which is the conormal bundle of $S$ in $\tilde{S}$, in particular, a line bundle.

Lemma 1.5. Let $S$ be a rational normal scroll and $\tilde{S}$ a carpet whose reduced part is $S$, and let $\mathcal{L}$ be the dual of the ideal sheaf defining $S$ in $\tilde{S}$. Then $\tilde{S}$ is a K3 carpet if and only if $\mathcal{L} \simeq \omega_{S}{ }^{*}$.

Proof. First assume $\mathcal{L} \simeq \omega_{S}^{*}$, so we have an exact sequence

$$
0 \rightarrow \omega_{S} \rightarrow \mathcal{O}_{\tilde{S}} \rightarrow \mathcal{O}_{S} \rightarrow 0 .
$$


From $\mathrm{H}^{1}\left(\mathcal{O}_{S}\right)=\mathrm{H}^{1}\left(\omega_{S}\right)=0$ it follows that $\mathrm{H}^{1}\left(\mathcal{O}_{\tilde{S}}\right)=0$. If we apply to (1.5.1) the

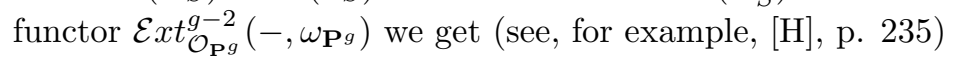

$$
0 \rightarrow \omega_{S} \rightarrow \omega_{\tilde{S}} \rightarrow \mathcal{O}_{S} \rightarrow 0 .
$$

Since $\mathrm{H}^{1}\left(\omega_{S}\right)=0$, the map $\mathrm{H}^{0}\left(\omega_{\tilde{S}}\right) \rightarrow \mathrm{H}^{0}\left(\mathcal{O}_{S}\right)$ is an epimorphism. Therefore $1 \in \mathrm{H}^{0}\left(\mathcal{O}_{S}\right)$ can be lifted to $\mathrm{H}^{0}\left(\omega_{\tilde{S}}\right)$, and hence $\omega_{\tilde{S}} \simeq \mathcal{O}_{\tilde{S}}=0$.

Now assume $\tilde{S}$ is a K3 carpet. Apply the functor $\mathcal{E}_{x} t_{\mathcal{O}_{\mathbf{p}} g}^{g-2}\left(-, \omega_{\mathbf{P}^{g}}\right)$ to the exact sequence

$$
0 \rightarrow \mathcal{I} \rightarrow \mathcal{O}_{\tilde{S}} \rightarrow \mathcal{O}_{S} \rightarrow 0
$$

to obtain

$$
0 \rightarrow \omega_{S} \rightarrow \omega_{\tilde{S}} \rightarrow \mathcal{I}^{*} \otimes \omega_{S} \rightarrow 0 .
$$

If we tensor (1.5.2) with $\mathcal{O}_{S}$ and use the fact that $\omega_{\tilde{S}} \simeq \mathcal{O}_{\tilde{S}}$, we get a surjection $\mathcal{O}_{S} \rightarrow \mathcal{I}^{*} \otimes \omega_{S}$. Thus $\mathcal{I}^{*} \otimes \omega_{S} \simeq \mathcal{O}_{S}$ and $\mathcal{I} \simeq \omega_{S}$.

(1.6) Lemmas 1.4 and 1.5 imply that in order to see how many K3 carpets are supported in a particular rational normal scroll $S$, one has to compute how many bundle inclusions there are from $\omega_{S}^{*}$ into $\mathcal{N}_{S / \mathbf{P}^{g}}$, or equivalently, how many nowhere vanishing sections there are in $\mathrm{H}^{0}\left(\mathcal{N}_{S / \mathbf{P} g} \otimes \omega_{S}\right)$. Therefore Theorem 1.3 follows from the following:

Proposition 1.7. Let $S=S(a, b), a \geq b$, be the rational normal scroll that corresponds to the embedding of $\mathbf{P}(\mathcal{E})$ into $\mathbf{P}^{N}$ by $\mathcal{O}_{\mathbf{P}(\mathcal{E})}(1)$, where $\mathcal{E}=\mathcal{O}_{\mathbf{P}^{1}}(a) \oplus \mathcal{O}_{\mathbf{P}^{1}}(b)$. Let $\omega$ be the canonical bundle of $\mathbf{P}(\mathcal{E})$. Then $\mathrm{H}^{0}\left(\mathcal{N}_{S / \mathbf{P}^{N}} \otimes \omega\right)=\mathbf{C}\langle s\rangle$, where $s$ is a nowhere vanishing section, and $\mathrm{H}^{1}\left(\mathcal{N}_{S / \mathbf{P}^{N}} \otimes \omega\right)=\mathrm{H}^{2}\left(\mathcal{N}_{S / \mathbf{P}^{N}} \otimes \omega\right)=0$.

Proof. We use the exact sequence

$$
\left.0 \rightarrow \mathcal{T}_{S} \otimes \omega \rightarrow \mathcal{T}_{\mathbf{P}^{N}}\right|_{S} \otimes \omega \rightarrow \mathcal{N}_{S / \mathbf{P}^{N}} \otimes \omega \rightarrow 0
$$

to compute $\pi_{*}\left(\mathcal{N}_{S / \mathbf{P}^{N}} \otimes \omega\right)$, where $\pi$ denotes the projection from $S$ to $\mathbf{P}^{1}$. To compute $\pi_{*}\left(\mathcal{T}_{S} \otimes \omega\right)$ we use the exact sequences

$$
0 \rightarrow \mathcal{T}_{S / \mathbf{P}^{1}} \otimes \omega \rightarrow \mathcal{T}_{S} \otimes \omega \rightarrow \pi^{*} \mathcal{T}_{\mathbf{P}^{1}} \otimes \omega \rightarrow 0
$$

and

$$
0 \rightarrow \Omega_{S / \mathbf{P}^{1}} \rightarrow \pi^{*} \mathcal{E} \otimes \mathcal{O}_{\mathbf{P}(\mathcal{E})}(-1) \rightarrow \mathcal{O}_{\mathbf{P}(\mathcal{E})} \rightarrow 0
$$

which is a relative version of the Euler sequence.

Let $\mathcal{E}^{\prime}=\mathcal{E} \otimes \mathcal{O}_{\mathbf{P}^{1}}(-a)=\mathcal{O}_{\mathbf{P}^{1}} \oplus \mathcal{O}_{\mathbf{P}^{1}}(b-a)$. Then $\mathcal{O}_{\mathbf{P}\left(\mathcal{E}^{\prime}\right)}(1)=\mathcal{O}\left(C_{0}\right)$, where $C_{0}$ denotes the minimal section of $\pi: S \rightarrow \mathbf{P}^{1}$. Therefore, by exact sequence (1.7.2) we obtain

$$
\Omega_{S / \mathbf{P}^{1}}=\bigwedge^{2}\left(\mathcal{O}\left(-C_{0}\right) \oplus \mathcal{O}\left(-C_{0}+(b-a) f\right)\right)=\mathcal{O}\left(-2 C_{0}+(b-a) f\right) .
$$

Also, we know that $\omega=\mathcal{O}\left(-2 C_{0}+(b-a-2) f\right)$ and that $\pi^{*} \mathcal{T}_{\mathbf{P}^{1}}=\mathcal{O}(2 f)$. Hence we obtain the sequence

$$
0 \rightarrow \mathcal{O}(-2 f) \rightarrow \mathcal{T}_{S} \otimes \omega \rightarrow \mathcal{O}\left(-2 C_{0}+(b-a) f\right) \rightarrow 0 .
$$

We apply $\pi_{*}$ and get

$$
\begin{aligned}
0 & \rightarrow \mathcal{O}_{\mathbf{P}^{1}}(-2) \rightarrow \pi_{*}\left(\mathcal{T}_{S} \otimes \omega\right) \rightarrow \pi_{*}\left(\mathcal{O}\left(-2 C_{0}+(b-a) f\right)=0\right. \\
& \rightarrow R^{1} \pi_{*} \mathcal{O}(-2 f)=0 \rightarrow R^{1} \pi_{*}\left(\mathcal{T}_{S} \otimes \omega\right) \rightarrow R^{1} \pi_{*}\left(\mathcal{O}\left(-2 C_{0}+(b-a) f\right) \rightarrow 0 .\right.
\end{aligned}
$$


Therefore $\pi_{*}\left(\mathcal{T}_{S} \otimes \omega\right)=\mathcal{O}_{\mathbf{P}^{1}}(-2)$ and

$$
R^{1} \pi_{*}\left(\mathcal{T}_{S} \otimes \omega\right)=R^{1} \pi_{*}\left(\mathcal{O}\left(-2 C_{0}+(b-a) f\right)=\left(\pi_{*} \mathcal{O}\right)^{*}=\mathcal{O}_{\mathbf{P}^{1}}\right.
$$

by relative Serre duality.

To compute $\pi_{*}\left(\left.\mathcal{T}_{\mathbf{P}^{N}}\right|_{S} \otimes \omega\right)$ we push forward the presentation of $\left.\mathcal{T}_{\mathbf{P}^{N}}\right|_{S} \otimes \omega$, which comes from the Euler sequence

$$
\left.0 \rightarrow \mathcal{O}\left(-2 C_{0}+(b-a-2) f\right) \rightarrow\left(\mathcal{O}\left(-C_{0}+(b-2) f\right)\right)^{\oplus(N+1)} \rightarrow \mathcal{T}_{\mathbf{P}^{N}}\right|_{S} \otimes \omega \rightarrow 0,
$$

and we obtain

$$
\begin{gathered}
0 \rightarrow \pi_{*}\left(-2 C_{0}+(b-a-2) f\right) \rightarrow \pi_{*}\left[\left(\mathcal{O}\left(-C_{0}+(b-2) f\right)\right)^{\oplus(N+1)}\right]=0 \\
\rightarrow \pi_{*}\left(\left.\mathcal{T}_{\mathbf{P}^{N}}\right|_{S} \otimes \omega\right) \rightarrow R^{1} \pi_{*}\left(-2 C_{0}+(b-a-2) f\right) \\
\rightarrow R^{1} \pi_{*}\left[\left(\mathcal{O}\left(-C_{0}+(b-2) f\right)\right)^{\oplus(N+2)}\right]=0 .
\end{gathered}
$$

Thus $R^{1} \pi_{*}\left(\left.\mathcal{T}_{\mathbf{P}^{N}}\right|_{S} \otimes \omega\right)=0$ and

$$
\pi_{*}\left(\left.\mathcal{T}_{\mathbf{P}^{N}}\right|_{S} \otimes \omega\right)=R^{1} \pi_{*}\left(-2 C_{0}+(b-a-2) f\right)=\left(\pi_{*} \mathcal{O}(2 f)\right)^{*}=\mathcal{O}_{\mathbf{P}^{1}}(-2),
$$

by relative Serre duality. Applying $\pi_{*}$ to (1.7.1), we get

$$
0 \rightarrow \mathcal{O}_{\mathbf{P}^{1}}(-2) \rightarrow \mathcal{O}_{\mathbf{P}^{1}}(-2) \rightarrow \pi_{*}\left(\mathcal{N}_{S / \mathbf{P}^{N}} \otimes \omega\right) \rightarrow \mathcal{O}_{\mathbf{P}^{1}} \rightarrow 0 .
$$

Hence

$$
\begin{aligned}
& \pi_{*}\left(\mathcal{N}_{S / \mathbf{P}^{N}} \otimes \omega\right)=\mathcal{O}_{\mathbf{P}^{1}}, \\
& R^{1} \pi_{*}\left(\mathcal{N}_{S / \mathbf{P}^{N}} \otimes \omega\right)=0 .
\end{aligned}
$$

This means that there exists a nonzero global section $s$ of $\mathcal{N}_{S / \mathbf{P}^{N}} \otimes \omega$. This section cannot vanish identically at any fiber of $\pi$. But the fibers of $\pi$ are projective lines, and hence, by (1.7.3), the restriction of $\mathcal{N}_{S / \mathbf{P}^{N}} \otimes \omega$ to a fiber is isomorphic to $\mathcal{O}_{\mathbf{P}^{1}} \oplus \mathcal{F}$ for some vector bundle $\mathcal{F}$ without global sections. This implies that the restriction of $s$ to each fiber is nowhere vanishing. This proves the statement about $\mathrm{H}^{0}\left(\mathcal{N}_{S / \mathbf{P}^{N}} \otimes \omega\right)$. The statement about $\mathrm{H}^{1}$ and $\mathrm{H}^{2}$ follows from (1.7.3) and (1.7.4).

\section{A CHARACTERIZATION FOR RIBBONS}

The next theorem gives a way to decide whether a scheme is a ribbon by using induction on the dimension.

Theorem 2.1. Let $D$ be an scheme such that $D_{\text {red }}$ is equidimensional. $D$ is a ribbon iff for every closed point $p \in D_{\text {red }}$ there exists $h_{p} \in \mathcal{O}_{D, p}$ such that $\mathcal{O}_{D, p} /\left(h_{p}\right)$ is the structure sheaf of a ribbon of dimension $\operatorname{dim} D-1$, whose reduced structure is $\mathcal{O}_{D_{\text {red }}, p} /\left(h_{p}\right)$.

In order to prove the theorem we will need the following:

Remark 2.1.1. Let $M$ be a module over a ring $A$. Let $a \in A$ be a non-zero-divisor for $M$. Then $\operatorname{Tor}_{A}^{1}(M, A /(a))=0$. For example, in the situation of Theorem 2.1, $h_{p}$ is non-zero-divisor for $\mathcal{O}_{D_{r e d}}$ and $\operatorname{Tor}^{1}\left(\mathcal{O}_{D_{\text {red }}}, \mathcal{O}_{D, p} /\left(h_{p}\right)\right)=0$.

(2.2) Proof of Theorem 2.1. The "only if" part is trivial. For the "if" part let $\mathcal{I}$ be the ideal sheaf of $D_{\text {red }}$ in $D$. We have to show that $\mathcal{I} / \mathcal{I}^{2}$ is a locally free sheaf over $\mathcal{O}_{D_{\text {red }}}$ and that $\mathcal{I}^{2}=0$.

Step $1\left(\mathcal{I} / \mathcal{I}^{2}\right.$ is locally free). 
Let us fix a closed point $p \in D_{\text {red }}$. The ideal $\left(\mathcal{I}_{p}+h_{p}\right) /\left(h_{p}\right)=\mathcal{J}_{p}$ is the ideal of the reduced part of a ribbon, so $\mathcal{J}_{p} / \mathcal{J}_{p}^{2}=\mathcal{J}_{p}$ is a free module generated by one element. By Remark 2.1.1, $\mathcal{J}_{p}=\mathcal{I}_{p} \otimes \mathcal{O}_{D, p} /\left(h_{p}\right)$, and hence $\mathcal{I}_{p} / \mathcal{I}_{p}^{2} \otimes \mathcal{O}_{D_{\text {red }}, p} /\left(h_{p}\right)=$ $\mathcal{I}_{p} / \mathcal{I}_{p}^{2} \otimes \mathcal{O}_{D, p} /\left(h_{p}\right)=\mathcal{J}_{p} / \mathcal{J}_{p}^{2}$ is a free cyclic module. Thus, by Nakayama, $\mathcal{I}_{p} / \mathcal{I}_{p}^{2}$ is also a cyclic module over $\mathcal{O}_{D_{r e d}, p}$ generated by an element not vanishing at $p$. This is true for any closed point $p \in D_{\text {red }}$, i.e., the rank of $\mathcal{I} / \mathcal{I}^{2}$ is 1 for any closed point $p \in D_{\text {red }}$. Hence $\mathcal{I} / \mathcal{I}^{2}$ is locally free over $\mathcal{O}_{D_{\text {red }}}$.

Step $2\left(\mathcal{I}^{2}=0\right)$.

Fix again $p \in D_{\text {red }}$. We claim that $\mathcal{I}_{p}^{2} \otimes \mathcal{O}_{D, p} /\left(h_{p}\right)=0$. Indeed, as we remark in Step 1, $\left(\mathcal{I}_{p}+h_{p}\right) /\left(h_{p}\right)=\mathcal{J}_{p}$ is the ideal of the reduced part of a ribbon in its structure sheaf, so $\mathcal{J}_{p}^{2}=0$ and hence $\mathcal{I}_{p}^{2} \subset\left(h_{p}\right)$. This implies that $\mathcal{O}_{D, p} / \mathcal{I}_{p}^{2} \otimes$ $\mathcal{O}_{D, p} /\left(h_{p}\right)=\mathcal{O}_{D, p} /\left(h_{p}\right)$, so tensoring

$$
0 \rightarrow \mathcal{I}_{p}^{2} \rightarrow \mathcal{O}_{D, p} \rightarrow \mathcal{O}_{D, p} / \mathcal{I}_{p}^{2} \rightarrow 0
$$

by $\mathcal{O}_{D, p}$ we get

$$
\begin{gathered}
0 \rightarrow \operatorname{Tor}^{1}\left(\mathcal{O}_{D, p} / \mathcal{I}_{p}^{2}, \mathcal{O}_{D, p} /\left(h_{p}\right)\right) \rightarrow \mathcal{I}_{p}^{2} \otimes \mathcal{O}_{D, p} /\left(h_{p}\right) \\
\rightarrow \mathcal{O}_{D, p} /\left(h_{p}\right) \stackrel{\simeq}{\longrightarrow} \mathcal{O}_{D, p} /\left(h_{p}\right) \rightarrow 0 .
\end{gathered}
$$

In order to prove our claim, it suffices to prove that $\operatorname{Tor}^{1}\left(\mathcal{O}_{D, p} / \mathcal{I}_{p}^{2}, \mathcal{O}_{D, p} /\left(h_{p}\right)\right)=0$. If we tensor

$$
O \rightarrow \mathcal{I}_{p} / \mathcal{I}_{p}^{2} \rightarrow \mathcal{O}_{D, p} / \mathcal{I}_{p}^{2} \rightarrow \mathcal{O}_{D_{r e d}, p} \rightarrow 0
$$

by $\mathcal{O}_{D, p} /\left(h_{p}\right)$, we obtain

$$
\begin{gathered}
\operatorname{Tor}^{1}\left(\mathcal{I}_{p} / \mathcal{I}_{p}^{2}, \mathcal{O}_{D, p} /\left(h_{p}\right)\right) \rightarrow \operatorname{Tor}^{1}\left(\mathcal{O}_{D, p} / \mathcal{I}_{p}^{2}, \mathcal{O}_{D, p} /\left(h_{p}\right)\right) \\
\rightarrow \operatorname{Tor}^{1}\left(\mathcal{O}_{D_{\text {red }}}, \mathcal{O}_{D, p} /\left(h_{p}\right)\right)
\end{gathered}
$$

By Step 1 , we know that $\mathcal{I}_{p} / \mathcal{I}_{p}^{2}=\mathcal{O}_{D_{\text {red }}, p}$, so again by Remark 2.1.1, both $\operatorname{Tor}^{1}\left(\mathcal{O}_{D_{\text {red }}}, \mathcal{O}_{D, p} /\left(h_{p}\right)\right)$ and $\operatorname{Tor}^{1}\left(\mathcal{I}_{p} / \mathcal{I}_{p}^{2}, \mathcal{O}_{D, p} /\left(h_{p}\right)\right)$ vanish. Thus $\mathcal{I}_{p}^{2} \otimes \mathcal{O}_{D, p} /\left(h_{p}\right)=$ 0 , and by Nakayama's lemma, $\mathcal{I}_{p}^{2}=0$.

\section{Smoothings of K3 CARPets}

The purpose of this section is to prove the existence of smoothings of K3 carpets. By a smoothing of a K3 carpet we mean a flat family over a smooth curve with smooth generic fiber and with a special closed fiber isomorphic to the K3 carpet. We prove the result in two steps. Using the fact that rational normal scrolls $F_{0}, \ldots, F_{4}$ admit a generically $2: 1$ map from a hyperelliptic K3 surface, we construct, in a rather explicit way, smoothings of $\mathrm{K} 3$ carpets supported on $F_{0}, \ldots, F_{4}$. Then, in Theorem 3.6 we will see that the remaining K3 carpets lie in the closure of the locus parametrizing $\mathrm{K} 3$ carpets supported on $F_{0}, \ldots, F_{4}$. In order to prove these results we will need some auxiliary lemmas.

In this section, a smooth curve will mean either an algebraic smooth curve or the analytic disc $\Delta$.

Remark 3.1. Let $\mathcal{X}$ be a flat family over a smooth curve $T$. If $\phi: \mathcal{X} \rightarrow \mathcal{Z}$ is a morphism over $T$, then the image $\mathcal{Y}$ of $\phi$ is flat over $T$. 
Proof. Let $\pi$ be the morphism from $\mathcal{Y}$ to $T$. By assumption $\pi_{*} \phi_{*} \mathcal{O}_{\mathcal{X}}$ is flat over $\mathcal{O}_{T}$, and therefore $\pi_{*} \mathcal{O}_{\mathcal{Y}} \hookrightarrow \pi_{*} \phi_{*} \mathcal{O}_{\mathcal{X}}$ is a subsheaf of a torsion free sheaf on $T$, so it is itself torsion free and hence flat.

Lemma 3.2. Let $\mathcal{X}$ be a flat family of irreducible varieties over a smooth curve $T$. Let $\zeta$ be a relatively globally generated, invertible sheaf on $\mathcal{X}$. Let $\phi$ be the morphism from $\mathcal{X}$ to $\mathbf{P}_{T}^{n}$ induced by its relative complete linear series and let $\mathcal{Y}$ be the image of $\mathcal{X}$ by $\phi$. Assume that $\phi$ is an embedding outside the central fiber, and a finite morphism of degree 2 when restricted to the central fiber. Let $H$ be an hyperplane in $\mathbf{P}^{n}$. Then $(\mathcal{Y} \cap(H \times T))_{t}=\mathcal{Y}_{t} \cap(H \times\{t\})$ and $\mathcal{Y} \cap(H \times T)$ is flat over $T$.

Proof. The pullback of $H \times T$ is a Cartier divisor on $\mathcal{X}$ whose zero locus defines a flat family of divisors $\mathcal{X}^{\prime}$. Indeed, the only thing to be checked (cf. [H], III.9.8.5) is whether the pullback of $H \times T$ is defined by a non-zero-divisor at $\mathcal{O}_{\mathcal{X}_{t}, p}$, for all $t \in T$ and for all $p \in \mathcal{X}_{t}$. This is obvious, since $\mathcal{X}_{t}$ is reduced and irreducible, and $H$ does not contain $\phi_{t}\left(\mathcal{X}_{t}\right)$.

Now, the image of $\mathcal{X}^{\prime}$ by $\phi$ is a flat family by the previous observation. Hence, if we see that $\phi\left(\mathcal{X}^{\prime}\right)=\mathcal{Y} \cap(H \times T)$, we are done. We have to prove that the morphism $\mathcal{O}_{\mathcal{Y} \cap(H \times T)} \rightarrow \phi_{0_{*}} \mathcal{O}_{\mathcal{X}}$, obtained by tensoring $\mathcal{O}_{\mathcal{Y}} \hookrightarrow \phi_{*} \mathcal{O}_{\mathcal{X}}$ by $\mathcal{O}_{\mathbf{P}_{T}^{n}} / \mathcal{I}(H \times T)$, is injective. Consider the exact sequence

$$
0 \rightarrow \mathcal{O}_{\mathcal{Y}} \stackrel{\alpha}{\rightarrow} \phi_{*} \mathcal{O}_{\mathcal{X}} \rightarrow \mathcal{F} \rightarrow 0 .
$$

The rank of $\phi_{*} \mathcal{O}_{\mathcal{X}}$ is 1 outside $\phi\left(\mathcal{X}_{0}\right)$ and 2 at $\phi\left(\mathcal{X}_{0}\right)$. The injection $\alpha$ of $\mathcal{O}_{\mathcal{Y}}$ into $\phi_{*} \mathcal{O}_{\mathcal{X}}$ is given by a nowhere vanishing global section of $\phi_{*} \mathcal{O}_{\mathcal{X}}$; hence $\alpha$ is an injection at each fiber. From all this, it follows that $\mathcal{F}$ is supported at $\mathcal{Y}_{0}$ and has rank 1 at every closed point $y \in \phi\left(\mathcal{X}_{0}\right)$ (i.e., it is a line bundle on $\phi\left(\mathcal{X}_{0}\right)$ ). By hypothesis $\phi\left(\mathcal{X}_{0}\right)$ is an irreducible variety, so $H$ is locally a nonzero divisor at every point of $\phi\left(\mathcal{X}_{0}\right)$. Remark 2.2.1 implies that $\operatorname{Tor}^{1}\left(\mathcal{F}, \mathcal{O}_{\mathbf{P}_{T}^{n}} / \mathcal{I}(H \times T)\right)=0$, so $\mathcal{O}_{\mathcal{Y} \cap(H \times T)} \rightarrow \phi_{0_{*}} \mathcal{O}_{\mathcal{X}}$ is injective as required.

The fact that $(\mathcal{Y} \cap(H \times T))_{t}=\mathcal{Y}_{t} \cap(H \times\{t\})$ is obvious.

We recover as a corollary of Theorem 2.1 the following result of Fong:

Corollary 3.3 ([F], Theorem 1(i)). Let $\mathcal{C}$ be a flat family of smooth curves over a smooth curve $T$ such that its central fiber $C$ is a hyperelliptic curve and its generic fiber is a nonhyperelliptic curve. If $\mathcal{D}$ is the image in $\mathbf{P}_{T}^{2 g-2}$ of $\mathcal{C}$ by the relative complete linear series of $\omega_{\mathcal{C} / T}$, then the central fiber of $\mathcal{D}$ is a canonical ribbon, supported in the image of $C$ by the complete linear system of $\omega_{C}$.

Proof. Note first that, by Remark $3.1, \mathcal{D}$ is flat over $T$. Let $D$ be the central fiber of $\mathcal{D}$. We want to prove that $D$ is a canonical ribbon (recall that $D$ is not the image of the central fiber of $\mathcal{C}$ ). The degree of $D$ is $2 g-2$ and its arithmetic genus is $g$. The reduced part of $D, D_{\text {red }}$, is a rational normal curve, namely, the image of $C$. By Theorem 2.1, in order to see that $D$ is a ribbon we need to check that at every point p of $D_{\text {red }}$, we can choose a hyperplane $H_{p}$ passing through $p$ such that $H_{p} \cap D$ is isomorphic to $(g-1)$ copies of $\operatorname{Spec}(\mathbf{C}(\epsilon))$. To see this, choose $H_{p}$ through $p$ intersecting $D_{\text {red }}$ at $g-1$ distinct points. Lemma 3.2 tells that $H_{p} \cap D$ is the flat limit of a family of $(2 g-2)$ points. $H_{p} \cap D$ must be non-reduced everywhere. If not, a point of $H_{p} \cap D$ would be a smooth point of $D$, and the degree of $D$ would be equal to the degree of $D_{\text {red }}$, which is $g-1$. On the other hand, the degree of each component of $H_{p} \cap D$ must be less than or equal to two; otherwise there would be a reduced point in $H_{p} \cap D$. 
Proposition 3.4. Let $(\mathcal{X}, \zeta)$ be a flat family of polarized K3 surfaces of genus $g$ over the disc $T$, whose central fiber $\left(\mathcal{X}_{0}, \zeta_{0}\right)$ is a hyperelliptic polarized K3 surface. Assume furthermore that $\zeta_{t}$ is a very ample line bundle on $\mathcal{X}_{t}$, for all $t \neq 0$. If $\mathcal{Y}=\phi_{\zeta}(\mathcal{X}) \subset \mathbf{P}_{T}^{g}$, then the central fiber $\mathcal{Y}_{0}$ is a K3 carpet.

Proof. First, we prove that $\mathcal{Y}_{0}$ is a carpet. By Theorem 2.1 we only have to see that through every point $p \in\left(\mathcal{Y}_{0}\right)_{\text {red }}$ there exists a hyperplane $H_{p}$ such that $\mathcal{Y}_{0} \cap H_{p}$ is a ribbon supported on $\left(\mathcal{Y}_{0}\right)_{\text {red }} \cap H_{p}$. By Bertini's theorem, we can choose a (generic) hyperplane $H_{p}$ that passes through $p$ and whose intersection with $\left(\mathcal{Y}_{0}\right)_{\text {red }}$ is a smooth curve. $\left(\mathcal{Y}_{0}\right)_{\text {red }} \cap H_{p}$ is a rational normal curve. By Remark 3.1 we know that $\mathcal{Y}$ is flat over $T$. By Lemma 3.2 we know that $\mathcal{Y}_{0} \cap H_{p}$ is the limit of a family of canonical curves in $\mathbf{P}^{g-1}$, namely, the image of a family of curves whose central fiber is hyperelliptic (and whose general fiber is not), mapped by the complete linear series of the relative dualizing sheaf. Corollary 3.3 tells us that $\mathcal{Y}_{0} \cap H_{p}$ is actually a canonical ribbon supported on $\left(\mathcal{Y}_{0}\right)_{\text {red }} \cap H_{p}$.

Second, we prove that the canonical sheaf of $\mathcal{Y}_{0}$ is trivial and that the irregularity of $\mathcal{Y}_{0}$ is 0 . Since $\mathcal{Y}_{0}$ is the flat limit of a family of smooth K3 surfaces, $\mathcal{X}\left(\mathcal{O}_{\mathcal{Y}_{0}}\right)=2$ and therefore $\mathrm{h}^{2}\left(\mathcal{O}_{\mathcal{Y}_{0}}\right) \geq 1$. Thus, there exists a nonzero global section $s$ of $\omega_{\mathcal{Y}_{0}}$. We intend to show that $s$ is nowhere vanishing. We have the exact sequence

$$
0 \rightarrow \omega_{\left(\mathcal{Y}_{0}\right)_{\text {red }}} \rightarrow \omega_{\mathcal{Y}_{0}} \rightarrow \mathcal{I}^{*} \otimes \omega_{\left(\mathcal{Y}_{0}\right)_{\text {red }}} \rightarrow 0
$$

that comes from dualizing:

$$
0 \rightarrow \mathcal{I} \rightarrow \mathcal{O}_{\mathcal{Y}_{0}} \rightarrow \mathcal{O}_{\left(\mathcal{Y}_{0}\right)_{\text {red }}} \rightarrow 0 .
$$

Therefore $\mathrm{H}^{0}\left(\omega_{\mathcal{Y}_{0}}\right)=\mathrm{H}^{0}\left(\mathcal{I}^{*} \otimes \omega_{\left(\mathcal{Y}_{0}\right)_{\text {red }}}\right)$. This implies that if $s$ vanishes at every closed point of $\left(\mathcal{Y}_{0}\right)_{\text {red }}$, then $s$ is the zero section. Thus, $Z(s) \varsubsetneqq\left(\mathcal{Y}_{0}\right)_{\text {red }}$. Assume it is not empty and take $p \in Z(s)$. In the first part of the proof we showed that the intersection $D$ of the generic hyperplane $H_{p}$ through $p$ with $\mathcal{Y}_{0}$ is a canonical ribbon in $H_{p}=\mathbf{P}^{g-1}$. This means that $\mathcal{O}_{D}(1)=\omega_{D}$. From the adjunction formula we obtain that $\left.\omega_{\mathcal{Y}_{0}}\right|_{D}=\mathcal{O}_{D}$. Hence $\left.s\right|_{D} \in \mathrm{H}^{0}\left(\mathcal{O}_{D}\right)$, and since $s$ vanishes at $p \in D_{\text {red }}$, $\left.s\right|_{D_{\text {red }}}$ must be the zero section; but this contradicts the fact that $Z(s) \varsubsetneqq\left(\mathcal{Y}_{0}\right)_{\text {red }}$. Therefore $Z(s)=\emptyset$ and $\omega_{\mathcal{Y}_{0}} \simeq \mathcal{O}_{\mathcal{Y}_{0}}$. Using $\mathcal{X}\left(\mathcal{O}_{\mathcal{Y}_{0}}\right)=2$, it follows that $\mathrm{h}^{1}\left(\mathcal{O}_{\mathcal{Y}_{0}}\right)=$ 0 .

We will use Proposition 3.4 to prove our main

Theorem 3.5. Any K3 carpet can be smoothed.

Before we prove Theorem 3.5 we need to know the stratification of the locus of K3 carpets:

Theorem 3.6. The scheme $U$ parametrizing (smooth) rational normal scrolls embeds into the Hilbert scheme of numerical K3 surfaces of degree $2 g-2$ in $\mathbf{P}^{g}$. The image of $U$ by this embedding parametrizes the K3 carpets in $\mathbf{P}^{g}$. In particular, K3 carpets supported on $S(a-1, b+1)$ lie on the closure of the locus of the Hilbert scheme parametrizing $K 3$ carpets supported on $S(a, b)$.

We use two propositions to prove Theorem 3.6. The first is a relative version of Theorem 1.3:

Proposition 3.7. Let $U$ be a smooth variety. If $p: \mathcal{S} \rightarrow U$ is a flat family of rational normal scrolls inside $\mathbf{P}_{U}^{g}$, then there exists a unique family $\tilde{\mathcal{S}}$ over $U$, whose fibers are K3 carpets and such that $\tilde{\mathcal{S}}_{\text {red }}=\mathcal{S}$. 
Proof. Let $\mathcal{N}$ be the normal bundle of $\mathcal{S}$ inside $\mathbf{P}_{U}^{g}$ and let $\omega$ denote the relative dualizing sheaf of $\mathcal{S} / U$, which is in this case a line bundle. By Proposition 1.7, $p_{*}(\mathcal{N} \otimes \omega)$ is also a line bundle. We claim that $\mathcal{N} \otimes \omega \otimes p^{*}\left(p_{*}(\mathcal{N} \otimes \omega)\right)^{*}$ has a nowhere vanishing section $s$ and that $\mathrm{H}^{0}\left(\mathcal{N} \otimes \omega \otimes p^{*}\left(p_{*}(\mathcal{N} \otimes \omega)\right)^{*}\right)=s \cdot \mathrm{H}^{0}\left(\mathcal{O}_{U}\right)$. Indeed, by projection formula,

$$
\begin{gathered}
\mathrm{H}^{0}\left(\mathcal{N} \otimes \omega \otimes p^{*}\left(p_{*}(\mathcal{N} \otimes \omega)\right)^{*}\right) \\
=\mathrm{H}^{0}\left(p_{*}\left(\mathcal{N} \otimes \omega \otimes p^{*}\left(p_{*}(\mathcal{N} \otimes \omega)\right)^{*}\right)=\mathrm{H}^{0}\left(\mathcal{O}_{U}\right),\right.
\end{gathered}
$$

and $1 \in \mathrm{H}^{0}\left(\mathcal{O}_{U}\right)$ corresponds to a section $s$ of $\mathrm{H}^{0}\left(\mathcal{N} \otimes \omega \otimes p^{*}\left(p_{*}(\mathcal{N} \otimes \omega)\right)^{*}\right)$ which does not vanish identically along any fiber of $p$. Since $\mathrm{H}^{0}\left(\mathcal{N}_{\mathcal{S}_{u} / \mathbf{P}^{g}} \otimes \omega_{\mathcal{S}_{u}}\right)=\mathbf{C} \cdot s^{\prime}$ by Proposition 1.5, where $s^{\prime}$ is a nowhere vanishing section, it follows that $s$ is nowhere vanishing. In particular, any nowhere vanishing section of $\mathcal{N} \otimes \omega \otimes p^{*}\left(p_{*}(\mathcal{N} \otimes \omega)\right)^{*}$ is a multiple of $s$ by a global section of $\mathcal{O}_{U}^{*}$. By Lemma 1.4, $s$ defines a double structure $\tilde{\mathcal{S}}$ on $\mathcal{S}$, and by the previous observation any other nowhere vanishing section of $\mathcal{N} \otimes \omega \otimes p^{*}\left(p_{*}(\mathcal{N} \otimes \omega)\right)^{*}$ defines the same double structure. The ideal sheaf of $\mathcal{S}$ in $\mathcal{O}_{\tilde{\mathcal{S}}}$ is the line bundle $\omega \otimes p^{*}\left(p_{*}(\mathcal{N} \otimes \omega)\right)^{*}$. Hence, since $\mathcal{S}$ is flat over $U$, it follows that $\tilde{\mathcal{S}}$ is also flat over $U$. This implies that $\tilde{\mathcal{S}}$ is a family of K3 carpets.

Now we prove the uniqueness of $\tilde{\mathcal{S}}$. Let $\tilde{\mathcal{S}}^{\prime}$ be a flat family over $U$ whose fibers $\tilde{\mathcal{S}}_{u}^{\prime}$ are K3 carpets such that $\left(\tilde{\mathcal{S}}_{u}^{\prime}\right)_{\text {red }}=\mathcal{S}_{u}$ for all $u \in U$. Using Theorem 2.1 inductively (we lift a regular sequence defining the point $u$ in $\mathcal{O}_{u, U}$ to $\mathcal{O}_{x, \tilde{\mathcal{S}}^{\prime}}$, where $x$ is any point in the inverse image of the morphism from $\tilde{\mathcal{S}}^{\prime}$ to $U$ ), we conclude that $\tilde{\mathcal{S}}^{\prime}$ is a double structure on $\mathcal{S}$. This is equivalent to the data of a vector bundle surjection $\mathcal{N}^{*} \rightarrow \mathcal{L} \rightarrow 0$, where $\mathcal{L}$ is a line bundle. By flatness and because $\tilde{\mathcal{S}}^{\prime}$ is a family of $\mathrm{K} 3$ carpets, we obtain that $\left.\mathcal{L}\right|_{\mathcal{S}_{u}}=\omega_{\mathcal{S}_{u}}$ for all $u \in U$. Therefore $\left.\left(\mathcal{L} \otimes \omega^{*}\right)\right|_{\mathcal{S}_{u}}=\mathcal{O}_{\mathcal{S}_{u}}$ and $p_{*}\left(\mathcal{L} \otimes \omega^{*}\right)$ is a line bundle. Moreover, $p^{*} p_{*}\left(\mathcal{L} \otimes \omega^{*}\right) \rightarrow$ $\mathcal{L} \otimes \omega^{*}$ is a surjective morphism of line bundles, and hence an isomorphism. Thus $\mathcal{N} \otimes \mathcal{L}=\mathcal{N} \otimes \omega \otimes p^{*} p_{*}\left(\mathcal{L} \otimes \omega^{*}\right)$. By hypothesis $\mathrm{H}^{0}(\mathcal{N} \otimes \mathcal{L})$ contains a nowhere vanishing section; hence $p_{*}(\mathcal{N} \otimes \mathcal{L})=\mathcal{O}_{U}$. By the projection formula it follows that $p_{*}\left(\mathcal{L} \otimes \omega^{*}\right)=\left(p_{*}(\mathcal{N} \otimes \omega)\right)^{*}$ and $\mathcal{N} \otimes \mathcal{L}=\mathcal{N} \otimes \omega \otimes p^{*}\left(p_{*}(\mathcal{N} \otimes \omega)\right)^{*}$. This implies that $\tilde{\mathcal{S}}^{\prime}=\tilde{\mathcal{S}}$.

(3.8) Recall that (smooth) rational normal scrolls are parametrized by a reduced, open subscheme $U$ of the Hilbert scheme (see, e.g., [A]). The subscheme $U$ is stratified as follows (see $[\mathrm{A}]$ or $[\mathrm{Ha}])$ : the scrolls of type $S(a+1, b-1)$, less balanced, lie on the closure of the locus parametrizing scrolls of type $S(a, b)$, more balanced (recall that $a \geq b$ ).

Proposition 3.9. Let $S$ be a rational normal scroll in $\mathbf{P}^{N}$. The dimension of $\mathrm{H}^{0}\left(\mathcal{N}_{S / \mathbf{P}^{N}}\right)$ is $(N+1)^{2}-7$, and $\mathrm{H}^{1}\left(\mathcal{N}_{S / \mathbf{P}^{N}}\right)$ and $\mathrm{H}^{2}\left(\mathcal{N}_{S / \mathbf{P}^{N}}\right)$ vanish.

Proof. The statement follows from the exact sequence presenting $\mathcal{N}_{S / \mathbf{P}^{N}}$, from the Euler sequence on $\mathbf{P}^{N}$, and from the sequence relating the tangent bundle of $S$, the relative tangent bundle of the fibration to $\mathbf{P}^{1}$ and the pullback of the tangent bundle to $\mathbf{P}^{1}$.

(3.10) Proof of Theorem 3.6. The scheme $U$ is smooth (by Proposition 3.9 and $[\mathrm{S}]$, Corollaries 8.5 and 8.6; see also [A]). Thus, we can apply Proposition 3.7, and by the universal property of the Hilbert scheme, we obtain a morphism $\varphi$ from $U$ to the Hilbert scheme of numerical K3 surfaces. Let $Z$ be the image of $\varphi$. The scheme $Z$ parametrizes the K3 carpets inside the Hilbert scheme. To see that $\varphi$ 
is an isomorphism onto $Z$ it suffices, since both $U$ and $Z$ are varieties and we are working over $\mathbf{C}$, to show that there exists a morphism $\Psi$ that is a set-theoretical inverse of $\varphi$. To construct $\Psi$, consider the pull-back to $Z$ of the universal family on the Hilbert scheme. The fibers of this pull-back are K3 carpets. If we take the reduced structure of the pullback, we end up with a family of rational normal scrolls over $Z$. The universal property of the Hilbert scheme gives us the existence of $\Psi$. (3.8).

The observation about the stratification of the locus of K3 carpets follows from

(3.11) Proof of Theorem 3.5. First consider the K3 carpets whose reduced structure is a rational normal scroll $F$ (embedded in $\mathbf{P}^{g}$ as a variety of minimal degree) of type $F_{0}, \ldots, F_{4}$. The scroll $F$ can be realized as the image of the morphism induced by the hyperelliptic linear series of a polarized hyperelliptic $\mathrm{K} 3$ surface $(X, L)$. We give here a sketch of the construction of $(X, L)$; for more details, see $[\mathrm{D}]$ or $[\mathrm{R}]$. Take a curve $C$ in $\left|-2 K_{F}\right|$ with at worst certain mild singularities. Then the desingularization $X$ of the double cover of $F$ ramified along $C$ is a K3 surface. The line bundle $L$ is the pullback of $\mathcal{O}_{F}(1)$. Let $E$ be the elliptic pencil obtained as pullback of the ruling of $F$. In this situation the Picard lattice of $(X, L)$ contains a sublattice generated by $L$ and by $E$ with intersection matrix

$$
\left(\begin{array}{cc}
2 g-2 & 2 \\
2 & 0
\end{array}\right)
$$

Using the fact that the space of periods is a fine moduli space for polarized, marked $\mathrm{K} 3$ surfaces (see $[\mathrm{SP}]$ ), one can find a family $(\mathcal{X}, \zeta)$ of polarized K3 surfaces over the analytic disc $T$, whose central fiber $\left(\mathcal{X}_{0}, \zeta_{0}\right)$ is isomorphic to $(X, L)$ and such that $\zeta_{t}$ is very ample if $t \neq 0$. This is achieved by taking a path in the period space in this way: the central point corresponds to a period containing $E$, and the other points correspond to periods containing neither $E$ nor any class with nonpositive intersection with $L$. Let $\mathcal{Y}$ be $\phi_{\zeta}(\mathcal{X}) \subset \mathbf{P}_{T}^{g}$. Proposition 3.4 tells us that there exists a $\mathrm{K} 3$ carpet structure on $F$ that can be smooth, namely, $\mathcal{Y}_{0}$. This proves the theorem in this case, since we know by Theorem 1.3 that there is a unique K3 carpet structure on any given rational normal scroll.

We have just proven that $\mathrm{K} 3$ carpets on rational normal scrolls of type $F_{0}, \ldots, F_{4}$ lie on the closure of at least one component parametrizing smooth K3 surfaces in the Hilbert scheme. By Theorem 3.6, the remaining K3 carpets also lie in the closure of that (those) component(s).

\section{The Hilbert scheme near the Points of a K3 CARPet}

In this section we study the geometry of the Hilbert scheme of numerical K3 surfaces (i.e., regular subschemes of projective space with trivial dualizing sheaf) at the locus parametrizing K3 carpets. We start by settling the question of whether the Hilbert points of the K3 carpets are smooth.

Theorem 4.1. Let $\tilde{S}$ be a K3 carpet supported on $S=S(a, b)$, where $a \geq b$ and $g=a+b$. The Hilbert point of $\tilde{S}$ is nonsingular iff $0 \leq a-b \leq 2$.

Proof. We have proved in Theorem 3.5 that K3 carpets are smoothable. Since the dimension of a component parametrizing smooth K3 surfaces is $\operatorname{dim} \operatorname{PGL}(g)+19=$ $(g+1)^{2}+18$, a K3 carpet represents a smooth point of the Hilbert scheme iff 
$\mathrm{h}^{0}\left(\mathcal{N}_{\tilde{S} / \mathbf{P}^{g}}\right)=(g+1)^{2}+18$. To compute the cohomology of $\mathcal{N}_{\tilde{S} / \mathbf{P}^{g}}$ we tensor the sequence

$$
0 \rightarrow \omega_{S} \rightarrow \mathcal{O}_{\tilde{S}} \rightarrow \mathcal{O}_{S} \rightarrow 0
$$

by $\mathcal{N}_{\tilde{S} / \mathbf{P}^{g}}$. Since $\tilde{S}$ is locally a complete intersection, the sheaf $\mathcal{N}_{\tilde{S} / \mathbf{P}^{g}}$ is a vector bundle and we obtain

$$
0 \rightarrow \mathcal{N}_{\tilde{S} / \mathbf{P}^{g}} \otimes \omega_{S} \rightarrow \mathcal{N}_{\tilde{S} / \mathbf{P}^{g}} \rightarrow \mathcal{N}_{\tilde{S} / \mathbf{P}^{g}} \otimes \mathcal{O}_{S} \rightarrow 0
$$

Thus, we have

$$
\chi\left(\mathcal{N}_{\tilde{S} / \mathbf{P}^{g}}\right)=\chi\left(\mathcal{N}_{\tilde{S} / \mathbf{P}^{g}} \otimes \omega_{S}\right)+\chi\left(\mathcal{N}_{\tilde{S} / \mathbf{P}^{g}} \otimes \mathcal{O}_{S}\right) .
$$

Let $\mathcal{I}_{S}$ (respectively $\mathcal{I}_{\tilde{S}}$ ) be the ideal of $S$ (respectively $\tilde{S}$ ) in $\mathbf{P}^{g}$. Since $\mathcal{I}_{\tilde{S}} / \mathcal{I}_{\tilde{S}}^{2}$ is a bundle, taking its dual and restricting it to $S$ commute. Hence,

$$
\begin{gathered}
\mathcal{N}_{\tilde{S} / \mathbf{P}^{g}} \otimes \mathcal{O}_{S}=\mathcal{H o m}_{\tilde{S}}\left(\mathcal{I}_{\tilde{S}} / \mathcal{I}_{\tilde{S}}^{2}, \mathcal{O}_{\tilde{S}}\right) \otimes \mathcal{O}_{S} \\
=\mathcal{H o m}_{S}\left(\mathcal{I}_{\tilde{S}} / \mathcal{I}_{\tilde{S}}^{2} \otimes \mathcal{O}_{S}, \mathcal{O}_{S}\right)=\mathcal{H o m}_{S}\left(\mathcal{I}_{\tilde{S}} / \mathcal{I}_{\tilde{S}} \mathcal{I}_{S}, \mathcal{O}_{S}\right) .
\end{gathered}
$$

Therefore $\mathcal{N}_{\tilde{S} / \mathbf{P}^{g}} \otimes \mathcal{O}_{S}$ sits in the sequence

$$
0 \rightarrow \mathcal{H o m}_{S}\left(\mathcal{I}_{\tilde{S}} / \mathcal{I}_{S}^{2}, \mathcal{O}_{S}\right) \rightarrow \mathcal{N}_{\tilde{S} / \mathbf{P}^{g}} \otimes \mathcal{O}_{S} \rightarrow \mathcal{Q} \rightarrow 0
$$

From the sequence

$$
0 \rightarrow \mathcal{I}_{\tilde{S}} / \mathcal{I}_{S}^{2} \rightarrow \mathcal{I}_{S} / \mathcal{I}_{S}^{2} \rightarrow \mathcal{I}_{S} / \mathcal{I}_{\tilde{S}} \rightarrow 0
$$

we see at once that $\mathcal{Q}$ is a line bundle. We claim that $\mathcal{Q}=\omega_{S}^{-2}$. From (4.1.3) it follows that $\mathcal{Q}=\bigwedge^{g-2}\left(\mathcal{N}_{\tilde{S} / \mathbf{P}^{g}} \otimes \mathcal{O}_{S}\right) \otimes \bigwedge^{g-3} \mathcal{I}_{\tilde{S}} / \mathcal{I}_{S}^{2}$. Dualizing sequence (4.1.4) and taking the wedge, we obtain that

$$
\bigwedge^{g-3} \mathcal{I}_{\tilde{S}} / \mathcal{I}_{S}^{2}=\omega_{S}^{*} \otimes \bigwedge^{g-2} \mathcal{N}_{S / \mathbf{P}^{g}}^{*}=\omega_{S}^{-2} \otimes \mathcal{O}_{S}(-g-1)
$$

Using adjunction and the fact that $\tilde{S}$ is a K3 carpet, it follows that $\bigwedge^{g-2} \mathcal{N}_{\tilde{S} / \mathbf{P} g}=$ $\mathcal{O}_{\tilde{S}}(g+1)$, and therefore $\bigwedge^{g-2}\left(\mathcal{N}_{\tilde{S} / \mathbf{P}^{g}} \otimes \mathcal{O}_{S}\right)=\mathcal{O}_{S}(g+1)$, so the claim is clear. Therefore we obtain the following exact sequences:

$$
\begin{aligned}
0 & \rightarrow \mathcal{H o m}_{S}\left(\mathcal{I}_{\tilde{S}} / \mathcal{I}_{S}^{2}, \mathcal{O}_{S}\right) \rightarrow \mathcal{N}_{\tilde{S} / \mathbf{P}^{g}} \otimes \mathcal{O}_{S} \rightarrow \omega_{S}^{-2} \rightarrow 0, \\
0 & \rightarrow \mathcal{H o m}_{S}\left(\mathcal{I}_{\tilde{S}} / \mathcal{I}_{S}^{2}, \mathcal{O}_{S}\right) \otimes \omega_{S} \rightarrow \mathcal{N}_{\tilde{S} / \mathbf{P}^{g}} \otimes \omega_{S} \rightarrow \omega_{S}^{*} \rightarrow 0,
\end{aligned}
$$

and from (4.1.4) we obtain

$$
\begin{aligned}
& 0 \rightarrow \omega_{S}^{*} \rightarrow \mathcal{N}_{S / \mathbf{P}^{g}} \rightarrow \mathcal{H o m}_{S}\left(\mathcal{I}_{\tilde{S}} / \mathcal{I}_{S}^{2}, \mathcal{O}_{S}\right) \rightarrow 0, \\
& 0 \rightarrow \mathcal{O}_{S} \rightarrow \mathcal{N}_{S / \mathbf{P}^{g}} \otimes \omega_{S} \rightarrow \mathcal{H o m}_{S}\left(\mathcal{I}_{\tilde{S}} / \mathcal{I}_{S}^{2}, \mathcal{O}_{S}\right) \otimes \omega_{S} \rightarrow 0 .
\end{aligned}
$$

Using (4.1.8) and Proposition 1.7, it follows that $\mathrm{H}^{1}\left(\mathcal{H o m}_{S}\left(\mathcal{I}_{\tilde{S}} / \mathcal{I}_{S}^{2}, \mathcal{O}_{S}\right) \otimes \omega_{S}\right)=0$. From (4.1.7) and Proposition 3.9, and the fact that $\mathrm{H}^{2}\left(\omega_{S}^{*}\right)=0$, it follows that $\mathrm{H}^{1}\left(\mathcal{H o m}_{S}\left(\mathcal{I}_{\tilde{S}} / \mathcal{I}_{S}^{2}, \mathcal{O}_{S}\right)\right)$ vanishes. Therefore

$$
\begin{gathered}
\mathrm{h}^{0}\left(\mathcal{N}_{\tilde{S} / \mathbf{P}^{g}} \otimes \mathcal{O}_{S}\right)=\mathrm{h}^{0}\left(\mathcal{H o m}_{S}\left(\mathcal{I}_{\tilde{S}} / \mathcal{I}_{S}^{2}, \mathcal{O}_{S}\right)\right)+\mathrm{h}^{0}\left(\omega_{S}^{-2}\right) \\
=\mathrm{h}^{0}\left(\mathcal{N}_{S / \mathbf{P}^{g}}\right)-\mathrm{h}^{0}\left(\omega_{S}^{*}\right)+\mathrm{h}^{1}\left(\omega_{S}^{*}\right)+\mathrm{h}^{0}\left(\omega_{S}^{-2}\right)
\end{gathered}
$$

and

$$
\begin{gathered}
\mathrm{h}^{0}\left(\mathcal{N}_{\tilde{S}, \mathbf{P}^{g}} \otimes \omega_{S}\right)=\mathrm{h}^{0}\left(\mathcal{H o m}_{S}\left(\mathcal{I}_{\tilde{S}} / \mathcal{I}_{S}^{2}, \mathcal{O}_{S}\right) \otimes \omega_{S}\right)+\mathrm{h}^{0}\left(\omega_{S}^{*}\right) \\
=\mathrm{h}^{0}\left(\mathcal{N}_{S / \mathbf{P}^{g}} \otimes \omega_{S}\right)-1+\mathrm{h}^{0}\left(\omega_{S}^{*}\right)=\mathrm{h}^{0}\left(\omega_{S}^{*}\right),
\end{gathered}
$$


by Proposition 1.7. By Proposition 3.7, $\mathrm{H}^{2}\left(\mathcal{N}_{\tilde{S} / \mathbf{P}^{g}}\right)=0$. From this and from (4.1.7) it follows that $\mathrm{H}^{2}\left(\mathcal{H o m}_{S}\left(\mathcal{I}_{\tilde{S}} / \mathcal{I}_{S}^{2}, \mathcal{O}_{S}\right)\right)$ vanishes. Using that $\mathrm{H}^{2}\left(\omega_{S}^{-2}\right)$ and $\mathrm{H}^{1}\left(\mathcal{H o m}_{S}\left(\mathcal{I}_{\tilde{S}} / \mathcal{I}_{S}^{2}, \mathcal{O}_{S}\right)\right)$ vanish, from (4.1.5) we obtain that $\mathrm{H}^{1}\left(\mathcal{N}_{\tilde{S} / \mathbf{P} g} \otimes \mathcal{O}_{S}\right)=$ $\mathrm{H}^{1}\left(\omega_{S}^{-2}\right)$ and that $\mathrm{H}^{2}\left(\mathcal{N}_{\tilde{S} / \mathbf{P}^{g}} \otimes \mathcal{O}_{S}\right)=0$. Analogously, from Proposition 1.7 and sequence (4.1.8) it follows that $\mathrm{H}^{2}\left(\mathcal{H o m}_{S}\left(\mathcal{I}_{\tilde{S}} / \mathcal{I}_{S}^{2}, \mathcal{O}_{S}\right) \otimes \omega_{S}\right)$ vanishes. Using that $\mathrm{H}^{2}\left(\omega_{S}^{*}\right)$ and $\mathrm{H}^{1}\left(\mathcal{H o m}_{S}\left(\mathcal{I}_{\tilde{S}} / \mathcal{I}_{S}^{2}, \mathcal{O}_{S}\right) \otimes \omega_{S}\right)$ vanish, from (4.1.6) we obtain that $\mathrm{H}^{1}\left(\mathcal{N}_{\tilde{S} / \mathbf{P}^{g}} \otimes \omega_{S}\right)=\mathrm{H}^{1}\left(\omega_{S}^{*}\right)$ and that $\mathrm{H}^{2}\left(\mathcal{N}_{\tilde{S} / \mathbf{P}^{g}} \otimes \omega_{S}\right)=0$. Therefore we can rewrite (4.1.2) as

$$
\begin{gathered}
\mathrm{h}^{0}\left(\mathcal{N}_{\tilde{S} / \mathbf{P}^{g}}\right)-\mathrm{h}^{1}\left(\mathcal{N}_{\tilde{S} / \mathbf{P}^{g}}\right)=\mathrm{h}^{0}\left(\mathcal{N}_{S / \mathbf{P}^{g}}\right)+\mathrm{h}^{0}\left(\omega_{S}^{-2}\right)-\mathrm{h}^{1}\left(\omega_{S}^{-2}\right) \\
=\mathrm{h}^{0}\left(\mathcal{N}_{S / \mathbf{P}^{g}}\right)+\chi\left(\omega_{S}^{-2}\right) .
\end{gathered}
$$

In Proposition 3.9 we showed that the dimension of $\mathrm{H}^{0}\left(\mathcal{N}_{S / \mathbf{P}^{g}}\right)$ is $(g+1)^{2}-7$. By Riemann-Roch one obtains that $\chi\left(\omega_{S}^{-2}\right)=25$. Thus, $\mathrm{h}^{0}\left(\mathcal{N}_{\tilde{S} / \mathbf{P}^{g}}\right)-\mathrm{h}^{1}\left(\mathcal{N}_{\tilde{S} / \mathbf{P}^{g}}\right)=$ $(g+1)^{2}+18$, and from this it follows that $\tilde{S}$ represents a nonsingular point of the Hilbert scheme iff $\mathrm{h}^{1}\left(\mathcal{N}_{\tilde{S} / \mathbf{P}^{g}}\right)=0$. From sequence (4.1.1) we get

$$
\mathrm{H}^{1}\left(\mathcal{N}_{\tilde{S} / \mathbf{P}^{g}} \otimes \omega_{S}\right) \rightarrow \mathrm{H}^{1}\left(\mathcal{N}_{\tilde{S} / \mathbf{P}^{g}}\right) \rightarrow \mathrm{H}^{1}\left(\mathcal{N}_{\tilde{S} / \mathbf{P}^{g}} \otimes \mathcal{O}_{S}\right) \rightarrow 0
$$

hence the key point is to compute the dimension of $\mathrm{H}^{1}\left(\mathcal{N}_{\tilde{S} / \mathbf{P}^{g}} \otimes \mathcal{O}_{S}\right)=\mathrm{H}^{1}\left(\omega_{S}^{-2}\right)$ and of $\mathrm{H}^{1}\left(\mathcal{N}_{\tilde{S} / \mathbf{P}^{g}} \otimes \omega_{S}\right)=\mathrm{H}^{1}\left(\omega_{S}^{*}\right)$. Pushing down to $\mathbf{P}^{1}$, we obtain that

$$
\mathrm{H}^{1}\left(\omega_{S}^{*}\right)=\mathrm{H}^{1}\left(\mathcal{O}_{\mathbf{P}^{1}}(a-b+2) \oplus \mathcal{O}_{\mathbf{P}^{1}}(2) \oplus \mathcal{O}_{\mathbf{P}^{1}}(b-a+2)\right)
$$

and that

$$
\mathrm{H}^{1}\left(\omega_{S}^{-2}\right)=\mathrm{H}^{1}\left(\bigoplus_{i=-2}^{2} \mathcal{O}_{\mathbf{P}^{1}}(i(b-a)+4)\right) .
$$

Therefore, if $0 \leq a-b \leq 2$, both $\mathrm{H}^{1}\left(\omega_{S}^{*}\right)$ and $\mathrm{H}^{1}\left(\omega_{S}^{-2}\right)$ are zero. Hence $\mathrm{H}^{1}\left(\mathcal{N}_{\tilde{S} / \mathbf{P}^{g}}\right)$ vanishes and $\tilde{S}$ corresponds to a nonsingular point of the Hilbert scheme. On the other hand, if $a-b>2$, the group $\mathrm{H}^{1}\left(\omega_{S}^{-2}\right)$ does not vanish and neither does $\mathrm{H}^{1}\left(\mathcal{N}_{\tilde{S} / \mathbf{P}^{g}}\right)$.

As a consequence of Theorem 4.1 we know that K3 carpets on rational normal scrolls of type $F_{0}, F_{1}, F_{2}$ belong only to one component of the Hilbert scheme of numerical K3 surfaces, and by Theorem 3.5 we know that the general point of that component is a smooth K3 surface. By using the smoothing constructed in (3.11) we are able to identify the component in question. The same construction allows us to prove that a K3 carpet contained in $\mathbf{P}^{g}$, when $g \equiv 1$ (4), and with reduced part isomorphic to the ruled surface $F_{4}$, belongs to two components of the Hilbert scheme. This fact provides a geometric explanation for the nonsmoothness of its Hilbert point.

Theorem 4.2. The K3 carpets supported on rational normal scrolls of type $F_{0}, F_{1}$ (and therefore any K3 carpet) belong to the "prime" component of the Hilbert scheme of numerical K3 surfaces.

Proof. Let $X$ be a hyperelliptic K3 surface mapping generically $2: 1$ to $F_{0}$ or $F_{1}$. If $X$ maps to $F_{0}$, the Picard group of $X$ contains a sublattice generated by two 
elliptic pencils $E_{1}$ and $E_{2}$. This sublattice has intersection matrix

$$
\left(\begin{array}{ll}
0 & 2 \\
2 & 0
\end{array}\right)
$$

If $X$ maps to $F_{1}$, the Picard group of $X$ contains a sublattice generated by an elliptic pencil $E$ and by a rational nodal curve $R$. This sublattice has intersection matrix

$$
\left(\begin{array}{cc}
0 & 2 \\
2 & -2
\end{array}\right)
$$

It is easy to check that these sublattices are primitive and, in particular, that $L_{n}=E_{i}+n E_{j}$ is primitive for all $n \geq 1$ and that $L_{n}=R+n E$ is primitive for all $n \geq 2$. The line bundles $L_{n}$ are the hyperelliptic line bundles which give a generically $2: 1$ map from $X$ to a rational normal scroll of type $F_{0}$ or $F_{1}$. Using the same reasoning as in (3.11) we can construct a family $(\mathcal{X}, \zeta)$ of polarized K3 surfaces whose central fiber is isomorphic to $\left(X, L_{n}\right)$ and whose general fiber $\left(\mathcal{X}_{t}, \zeta_{t}\right)$ is a K3 surface such that $\operatorname{Pic}\left(\mathcal{X}_{t}\right)$ is generated by $\zeta_{t}$. Therefore we can construct a smoothing of the K3 carpet supported on a rational normal scroll of type $F_{0}$ or $F_{1}$ such that the Picard group of the general fiber is generated by the hyperplane class.

Theorem 4.3. Let $g$ be greater than 9 and congruent to 1 modulo 4. Those K3 carpets inside $\mathbf{P}^{g}$ supported on a rational normal scroll $S$ of type $F_{4}$ belong to two components of the Hilbert scheme. One of them is the "prime" component. A general point of the other component corresponds to a smooth K3 surface with Picard number one but with hyperplane class divisible by two.

Proof. The Picard group of a hyperelliptic K3 surface $X$ mapping generically $2: 1$ to $F_{4}$ has a sublattice generated by an elliptic pencil $E$ and by a rational nodal curve $R$. This sublattice has intersection matrix

$$
\left(\begin{array}{cc}
0 & 1 \\
1 & -2
\end{array}\right)
$$

(see [D] for details). The hyperelliptic line bundles mapping $X$ generically $(2: 1)$ to a rational normal scroll of type $F_{4}$ are the line bundles $L_{n}=2 R+n E$ for all $n \geq 5$. If $n$ is even, the line bundle $L_{n}$ is not primitive, but the double of other line bundle. Therefore we can construct in that case a smoothing of $\tilde{S}$ with the following property: the general fiber has Picard number one but its hyperplane class does not generate the Picard group, but it is divisible by two. Thus the general fiber does not belong to the prime component. The hypothesis on $g$ being congruent to 1 modulo 4 comes in at this point, because in that case $n$ is even $\left(n=\frac{g+3}{2}\right)$.

We will devote the rest of the section to describing the deformation of K3 carpets to the union of two scrolls. Recall that the union of two rational normal scrolls of dimension 2 along a (reduced, but maybe reducible) elliptic curve, anticanonical with respect to both of them, has the numerical invariants of a K3 surface. Ciliberto, Lopez and Miranda prove in [CLM] that those unions of scrolls having smooth double locus (note that this condition forces the reducible K3 to be a union of two copies of $F_{0}, F_{1}$ or $F_{2}$ ) are smoothable. In fact, since any union of two rational normal scrolls along a reduced anticanonical curve can be deformed to a union of two scrolls with smooth double locus, it follows that any union of two scrolls along 
a reduced anticanonical elliptic curve is smoothable. Thus, which follows provides another, more indirect, proof of the smoothing of K3 carpets.

Theorem 4.4. The locus of K3 carpets lies on the closure of the locus parametrizing unions of two scrolls. Both loci lie on the closure of the open subscheme parametrizing smooth $K 3$ surfaces in the prime component.

Proof. Let $S$ be a rational normal scroll. Let $C$ be a curve in the linear equivalence class of the anticanonical divisor. The curve $C$ induces an embedding

$$
0 \rightarrow \mathcal{N}_{S, \mathbf{P}^{g}} \otimes \omega \rightarrow \mathcal{N}_{S, \mathbf{P}^{g}}
$$

and the image of the generator of $\mathrm{H}^{0}\left(\mathcal{N}_{S, \mathbf{P}^{g}} \otimes \omega\right)$ in $\mathrm{H}^{0}\left(\mathcal{N}_{S, \mathbf{P}^{g}}\right)$ corresponds to a first order deformation of $S$ in $\mathbf{P}^{g}$, keeping $C$ fixed. Since $\mathrm{h}^{1}\left(\mathcal{N}_{S, \mathbf{P}^{g}} \otimes \omega\right)=0$ by Proposition 1.7, this first order deformation extends to a deformation of $S$ over a smooth affine curve $U$, keeping $C$ fixed. We will call this deformation $\mathcal{S}_{1}$, and by an abuse of notation, we will denote its central fiber by $S$. Consider now another deformation $\mathcal{S}_{2}$ fixing $C$ (e.g., the trivial deformation $S \times U \subset \mathbf{P}_{U}^{g}$ ). The family $\mathcal{S}_{1} \cup \mathcal{S}_{2}$ is flat over $U$, and the general fiber is the union of two scrolls. We claim that the central fiber is a K3 carpet. Note that $\mathcal{S}_{1} \cap \mathcal{S}_{2}=S \cup(C \times T)$. For any point $x \in S$ we choose a hyperplane $H_{1}$ passing through $x$ such that $D:=S \cap H$ is a smooth rational normal curve and such that $\left(H_{1} \times U\right) \cap\left(\mathcal{S}_{1} \cap \mathcal{S}_{2}\right)$ is induced locally by a non-zero-divisor on $\mathcal{O}_{\mathcal{S}_{1} \cap \mathcal{S}_{2}}$. The scheme $\left(\mathcal{S}_{1} \cup \mathcal{S}_{2}\right) \cap\left(H_{1} \times U\right)$ is flat and equal to $\left(\mathcal{S}_{1} \cap\left(H_{1} \times U\right)\right) \cup\left(\mathcal{S}_{2} \cap\left(H_{1} \times U\right)\right)$. Denote $\mathcal{S}_{i} \cap\left(H_{1} \times U\right)$ by $\mathcal{S}_{i}^{\prime}$. Now $\mathcal{S}_{1}^{\prime} \cap \mathcal{S}_{2}^{\prime}=D \cup\left(\left(C \cap H_{1}\right) \times T\right)$, and through any point $y \in S \cap H_{1}$, we choose a hyperplane $H_{2}$ such that $D \cap H_{2}$ consists of distinct points and $\left(H_{2} \times U\right) \cap\left(\mathcal{S}_{1}^{\prime} \cap \mathcal{S}_{2}^{\prime}\right)$ is induced by a non-zero-divisor of $\mathcal{O}_{\mathcal{S}_{1}^{\prime} \cap \mathcal{S}_{2}^{\prime}}$. Again, the family $\left(\mathcal{S}_{1}^{\prime} \cup \mathcal{S}_{2}^{\prime}\right) \cap\left(H_{2} \times U\right)$ is flat and equal to $\left(\mathcal{S}_{1}^{\prime} \cap\left(H_{2} \times U\right)\right) \cup\left(\mathcal{S}_{2}^{\prime} \cap\left(H_{2} \times U\right)\right)$. The general fiber of $\left(\mathcal{S}_{1}^{\prime} \cup \mathcal{S}_{2}^{\prime}\right) \cap\left(H_{2} \times U\right)$ consists of $2 g-2$ distinct points, and the central fiber is supported on $g-1$ distinct points. Now the proof follows the same path as the proof of Proposition 3.4. By the same degree considerations, the central fiber of $\left(\mathcal{S}_{1}^{\prime} \cup \mathcal{S}_{2}^{\prime}\right) \cap\left(H_{2} \times U\right)$ is a 0 -dimensional ribbon. The central fiber of $\mathcal{S}_{1}^{\prime} \cup \mathcal{S}_{2}^{\prime}$ is also a ribbon, by Theorem 2.1 and Lemma 3.2. In fact, it is a canonical ribbon, because it is a nondegenerate ribbon of degree $2 g-2$ in $\mathbf{P}^{g-1}$. Again by Theorem 2.1 and Lemma 3.2, we obtain that the central fiber of $\mathcal{S}_{1} \cup \mathcal{S}_{2}$ is a carpet, and adjunction implies that it is a K3 carpet.

(4.5) An example of this degeneration can be constructed explicitly in the following way: let $S$ be a rational normal scroll in $\mathbf{P}^{g}, g \geq 4$. Let $C_{0}$ be the minimal section of $S$. Fix a smooth section $C^{\prime}$ not intersecting $C_{0}$. Let $\phi$ be the morphism from $C_{0}$ to $C^{\prime}$ defined by the fibers of $S$. Fix three points $a_{0}, b_{0}, c_{0}$ in $C_{0}$ and let $\phi\left(a_{0}\right)=a^{\prime}, \phi\left(b_{0}\right)=b^{\prime}$ and $\phi\left(c_{0}\right)=c^{\prime}$. Define $\phi_{x}: C_{0} \rightarrow C^{\prime}$ as the morphism that sends $a_{0}$ to $a^{\prime}, b_{0}$ to $b^{\prime}$ and $c_{0}$ to $x$. Let $(D, d)$ be a smooth projective curve and $f:(D, d) \rightarrow\left(C^{\prime}, c^{\prime}\right)$ a covering of $\left(C^{\prime}, c^{\prime}\right)$. Let $\Psi_{f}$ be defined as follows:

$$
\begin{aligned}
\Psi_{f}: C_{0} \times D & \rightarrow C^{\prime}, \\
(t, y) & \mapsto \phi_{f(y)}(t) .
\end{aligned}
$$

The morphism $\Psi_{f}$ defines a family $S_{f}$ of rational normal scrolls, parametrized by $D$. Each member of the family contains the reducible elliptic curve

$$
C:=C_{0} \cup C^{\prime} \cup \overline{a_{0} a^{\prime}} \cup \overline{b_{0} b^{\prime}}
$$


which is anticanonical in each scroll. Choosing $f_{1}, f_{2}:(D, d) \rightarrow\left(C^{\prime}, c^{\prime}\right), f_{1} \neq f_{2}$, we obtain $\mathcal{S}_{1}=S_{f_{1}}$ and $\mathcal{S}_{2}=S_{f_{2}}$ and a family $\mathcal{S}_{1} \cup \mathcal{S}_{2}$ as in the proof of 4.4. In fact, all this construction takes place inside the join $\Sigma$ of $C_{0}$ and $C^{\prime}$. The variety $\Sigma$ is a Fano threefold, i.e., some multiple of its anticanonical divisor $K_{\Sigma}$ is ample (this can be checked by looking at its desingularization).

When $a-b \leq 2$, the above construction fits into a more general one: consider a smooth elliptic normal curve $E$ in $\mathbf{P}^{g}$. Let $\Omega$ be its 2 -secant variety. The variety $\Omega$ is a "fake" Calabi-Yau 3-fold: its dualizing sheaf is trivial and the intermediate cohomology of its structure sheaf vanishes (see $[\mathrm{GP}]$ for details). On the other hand its desingularization is a projective bundle over $S_{2}(E)$; hence it has negative Kodaira dimension. It is singular along $E$. A $g_{2}^{1}$ on $E$ defines a rational normal scroll containing $E$ as a member of the anticanonical class. If we consider two families of $g_{2}^{1}$ 's specializing to a given one (the one defining the scroll on which our $\mathrm{K} 3$ carpet is supported), we obtain again a family as in the proof of 4.5. To go from this picture to the previous one, we just degenerate $E$ to $C$. The 3 -fold $\Omega$ degenerates to a reducible variety, one of whose components is $\Sigma$. Finally, we can identify the degenerations of the $g_{2}^{1}$ 's as pencils having degree 1 on $C_{0}$ and $C^{\prime}$ and degree 0 on $\overline{a_{0} a^{\prime}}$ and $\overline{b_{0} b^{\prime}}$. For example, in $\mathbf{P}^{4}$, the variety $\Omega$ is a quintic 3 -fold (in this case, since $\Omega$ is a hypersurface, one can easily check that it is "Calabi-Yau" in the above sense). The degeneration of $\Omega$ consists of $\Sigma$, which is a quadric cone, and three hyperplanes.

Observation 4.6. The K3 carpet supported on $S$ is a member of $\left|-K_{\Sigma}\right|$. However it is not possible to smooth it inside $\left|-K_{\Sigma}\right|$. In fact all members of $\left|-\omega_{\Sigma}\right|$ are singular. This follows from the fact that $\mathrm{H}^{0}\left(\omega_{\Sigma}^{*}\right)=\mathrm{H}^{0}\left(\omega_{C_{0}}^{*}\right) \otimes \mathrm{H}^{0}\left(\omega_{C^{\prime}}^{*}\right)$ (cf. [BE], $\S 8)$, which implies that an element of $\left|-K_{\Sigma}\right|$ is singular along both $C_{0}$ and $C^{\prime}$.

\section{REFERENCES}

[ACGH] E. Arbarello, M. Cornalba, P.A. Griffiths \& J. Harris, Geometry of Algebraic Curves, Vol. I, Springer, New York, 1985. MR 86h:14019

[A] P. Azcue, On the dimension of the Chow varieties, Harvard Thesis (1992).

[BE] D. Bayer \& D. Eisenbud, Ribbons and their canonical embeddings, Trans. Amer. Math. Soc. 347 (1995), 719-756. MR 95g:14032

[CLM] C. Ciliberto, A. Lopez \& R. Miranda, Projective degenerations of K3 surfaces, Gaussian maps, and Fano threefolds, Invent. Math. 114 (1993), 641-667. MR 94k:14028

[D] I. V. Dolgachev, On special algebraic K3 surfaces. I, Math. USSR Izvestija 7 (1973), 833-846.

[E] D. Eisenbud, Green's conjecture; an orientation for algebraists, Sundance 91: Proceedings of a Conference on Free Resolutions in Commutative Algebra and Algebraic Geometry, Jones and Barlett, 1992, pp. 51-78. MR 93e:13020

[EG] D. Eisenbud \& M. L. Green, Clifford indices of ribbons, Trans. Amer. Math. Soc. 347 (1995), 757-765. MR 95g:14033

[F] L.-Y. Fong, Rational ribbons and deformations of hyperelliptic curves, J. Algebraic Geom. 2 (1993), 295-307. MR 94c: 14020

[GP] M. Gross \& S. Popescu, Equations of $(1, d)$ polarized abelian surfaces, preprint.

[Ha] J. Harris, A bound on the geometric genus of projective varieties, Ann. Sci. Norm. Sup. Pisa (4) 8 (1981), 35-68. MR 82h:14010

[H] R. Hartshorne, Algebraic Geometry, Springer, Berlin, 1977. MR 57:3116

[HV] K. Hulek \& A. Van de Ven, The Horrocks-Mumford bundle and the Ferrand construction, Manuscripta Math. 50 (1985), 313-335. MR 86e:14005

[R] M. Reid, Hyperelliptic linear systems on a K3 surface, J. London Math. Soc. (2) 13 (1976), 427-437. MR 55:8044 
[SP] Séminaire Palaiseau, Géométrie des surfaces K3: modules et périodes, Astérisque 126, Soc. Math. France (1985). MR 87h:32052

[S] E. Sernesi, Topics on families of projective schemes, Queen's Papers in Pure and Appl. Math. 73, Queen's Univ., Kingston, Ont., 1986. MR 88b:14006

Departamento de Álgebra, Facultad de Matemáticas, Universidad Complutense de MADRID, 28040 MADrID, SpAIN

E-mail address: gallego@eucmos.sim.ucm.es

Department of Mathematics, Brandeis University, Waltham, Massachusetts 022549110

Current address: Department of Mathematics, Oklahoma State University, Stillwater, Oklahoma 74078

E-mail address: purna@littlewood.math.okstate.edu 